\title{
Advances in noise robust digit recognition using hybrid exemplar-based techniques
}

\author{
Jort F. Gemmeke and Hugo Van hamme \\ Department ESAT, KU Leuven, Belgium \\ \{Jort.Gemmeke, Hugo.Vanhamme\} desat.kuleuven.be
}

\begin{abstract}
Expressing noisy speech spectra as a linear combination of speech and noise exemplars has been shown to be a powerful tool to achieve noise robust ASR. Such a model has been used both to do feature enhancement (FE) and to directly provide noise robust speech state probabilities using a method called sparse classification (SC). The goal of this work is threefold: First, we integrate various SC advances recently proposed in literature, second, we improve upon the results obtained with FE through retraining and multi-condition training of the acoustic models used in the recognizer and finally, we propose the use of a single hybrid SC-FE system. In our experiments on AURORA-2 we obtain an impressive 3\% and 5\% average WER on matched and on mismatched noise types, respectively.

Index Terms: noise robustness, exemplar-based speech recognition, multi-stream decoding
\end{abstract}

\section{Introduction}

Even after decades of research, the accuracy of Automatic Speech Recognition (ASR) still rapidly degrades when speech is corrupted by noise. Even on relative simple tasks like digit recognition, for example the entry of telephone numbers, humans perform substantially better than noise robust ASR systems.

Many approaches to achieve noise robust ASR have been proposed; some of the better known techniques include spectral subtraction, Vector Taylor Series (VTS) approaches [1] and Missing Data techniques [2]. A class of methods that has gained recent prominence is based on compositional models: noisy speech spectra are represented as a linear, typically sparse, combination of basis atoms describing the individual speech and noise sources.

The use of compositional models has been employed in two ways to improve the robustness of ASR [3]. In both methods, first an optimal combination of basis atoms is found that jointly describes the noisy speech. In the first approach, the selected basis atoms pertaining to the clean speech are used to obtain a clean speech estimate, effectively doing feature enhancement (FE). In the second approach, dubbed Sparse Classification (SC) the basis atoms describing clean speech are associated with labels describing speech classes, such as phones or HMMstates. The weight of the selected speech atoms serves directly as evidence for the underlying speech classes, allowing recognition without further modelling by a Gaussian Mixture Model (GMM).

In previous work we proposed the use of exemplars, segments of audio extracted from a training corpus, as basis atoms. It was shown that both the FE approach and the SC approach improve the noise robustness, with SC yielding higher accuracies at low SNRs at the cost of lower accuracies at high SNRs [3]. Since then, many advances have been proposed to improve the speed, noise robustness on mismatched noise types, and clean speech accuracy of SC [4-6], but to date, these advances have not been combined into a single system. In this work, we incorporate all of the recently proposed advances for SC and investigate the cumulative improvements on SC and FE-based recognition that are obtained when these methods are combined.

In a second line of research, we investigate the impact of training GMM models on features that have been processed with $\mathrm{FE}$, and investigate the use of clean training data and multicondition training data. Finally, recent research has suggested that the relative lower accuracy of SC on clean speech can be improved by combination with GMM-based recognition in a multi-stream architecture [7]. Building on the recent successes with multi-stream approaches we propose to combine the FE approach and SC approach, both based on the same linear combination of basis atoms, into a single system.

Through experimentation on the well-known AURORA-2 database, we show the validity of our approach and finally obtain an impressive 3\% and 5\% average WER on matched and on mismatched noise types, respectively, using a multi-stream, multi-condition trained SC-FE recognizer. Comparisons with state-of-the art results published in literature reveal that these results are among the best ever published.

\section{Method}

\subsection{Compositional model for noise robust ASR}

The compositional model for noise robust ASR is based on representing observed noisy speech as a linear combination of speech and noise atoms. The atoms used for modeling noisy observations are $B \times T$ magnitude spectrogram segments, where $B$ is the number of Mel bands and $T$ is the number of consecutive frames in an atom. The collection of speech and noise atoms form a dictionary, and in this work speech and noise atoms are formed by exemplars, spectrogram segments extracted from a set of training utterances. With a noisy utterance $\mathbf{Y}$ represented in the form of overlapping windows of length $T$, a noisy spectrogram window $\Psi$ is reshaped to a vector $\psi$ and approximated as:

$$
\psi \approx\left[\mathbf{A}^{\mathrm{s}} \mathbf{A}^{\mathrm{n}}\right]\left[\begin{array}{l}
\mathbf{x}^{\mathrm{s}} \\
\mathbf{x}^{\mathrm{n}}
\end{array}\right]=\mathbf{A} \mathbf{x} \quad \text { s.t. } \quad \mathbf{x} \geq 0
$$

with $\mathrm{x}^{\mathrm{s}}$ and $\mathrm{x}^{\mathrm{n}}$ sparse linear combinations of the speech and noise dictionaries $\mathbf{A}^{\mathrm{s}}$ and $\mathbf{A}^{\mathrm{n}}$, respectively. The speech dictionary is formed by $J$ exemplars as its $E=B \cdot T$ dimensional columns, and the noise dictionary consists of $K$ atoms. The cost function to be minimized is composed of the KullbackLeibler divergence between $\psi$ and $\mathbf{A x}$, and the sum of $\mathbf{x}$ entries weighted element-wise by a sparsity penalty $\lambda[3]$. 
Table 1: WER obtained on AURORA-2 obtained with SC and FE recognition as a function of SNR. The settings described on each row are cumulative, i.e., the settings of the higher rows are also applied.

\begin{tabular}{|c|c|c|c|c|c|c|c|c|c|c|}
\hline \multirow[b]{3}{*}{ SNR $[\mathrm{dB}]$} & \multicolumn{5}{|c|}{ Sparse Classification } & \multicolumn{5}{|c|}{ Feature Enhancement } \\
\hline & \multirow[b]{2}{*}{ clean } & \multicolumn{2}{|c|}{ test set $A$} & \multicolumn{2}{|c|}{ test set $B$} & \multirow[b]{2}{*}{ clean } & \multicolumn{2}{|c|}{ test set $A$} & \multicolumn{2}{|c|}{ test set $B$} \\
\hline & & Avg 20-0 & -5 & Avg 20-0 & -5 & & Avg 20-0 & -5 & Avg 20-0 & -5 \\
\hline SC/FE Baseline & 5.4 & 10.6 & 42.8 & 14.4 & 62.2 & 0.2 & 6.3 & 41.7 & 10.2 & 64.1 \\
\hline Tuned sparsity & 4.2 & 8.1 & 39.1 & 12.1 & 60.8 & 0.2 & 5.6 & 40.4 & 9.6 & 62.2 \\
\hline 200 to 600 iterations & 4.0 & 7.3 & 35.2 & 11.1 & 58.5 & 0.2 & 4.6 & 33.8 & 8.5 & 59.4 \\
\hline 4000 to 10000 exemplars & 2.9 & 6.0 & 34.8 & 10.1 & 58.3 & 0.2 & 4.7 & 32.8 & 8.7 & 59.4 \\
\hline Artificial noise dictionary & 2.6 & 6.0 & 35.0 & 9.3 & 53.2 & 0.1 & 4.8 & 33.3 & 8.2 & 57.2 \\
\hline Noise sniffing & 2.6 & 5.9 & 35.2 & 8.2 & 47.0 & 0.1 & 4.8 & 33.1 & 7.2 & 53.3 \\
\hline
\end{tabular}

\subsection{Sparse classification and feature enhancement}

With each frame within each speech exemplar in the speech dictionary labelled using HMM-state labels obtained from a conventional GMM-based decoder, in SC the weights of the exemplars in the sparse representation $\mathbf{x}^{\mathrm{s}}$ are used to calculate estimates of state probabilities for each sliding window position [3]. We obtain a state probability $p_{\mathrm{SC}}\left(q_{t} \mid \mathbf{y}_{t}\right)$ for each state $q$ and for each frame $t$ of the observed noisy utterance $\mathbf{Y}$ by averaging the probabilities of overlapping windows. The utterance is then decoded by using a Viterbi search for the state sequences which maximize likelihood.

Alternatively, the activation matrix can be used for feature enhancement (FE). Two spectrogram estimates are derived from (1); a noisy speech reconstruction $\mathbf{A x}$, and a clean speech estimate $\mathbf{A}^{\mathrm{s}} \mathbf{x}^{\mathrm{s}}$. After resolving the overlapping windows through averaging, the element-wise speech-to-total ratio acts as a time-varying filter for the original noisy spectrogram $\mathbf{Y}$ [3]. Decoding is then done using a conventional GMM-based recognizer operating on acoustic features derived from the magnitude spectrograms representation.

\subsection{Multi-stream decoding}

In the hybrid exemplar-based GMM-based multi-stream approach, the state probabilities obtained with SC and with a GMM operating on FE-processed acoustic features are merged. Although both SC and FE employ the same dictionaries and the same sparse representation of the noisy speech, we assume the resulting state probabilities are sufficiently different to treat them as independent streams and write:

$$
p\left(q_{t} \mid y_{t}\right)=p_{\mathrm{SC}}\left(q_{t} \mid \mathbf{y}_{t}\right) p_{\mathrm{GMM}}\left(q_{t} \mid \mathbf{y}_{t}\right)
$$

with $p_{\mathrm{GMM}}$ the state probabilities obtained after evaluating the acoustic GMM model on FE-processed features and taking the state priors into account. To balance the two streams, we raise the SC and GMM state probabilities to a factor $\alpha$ and $\beta$, respectively.

\section{Experimental setup}

For our recognition experiments we used material from test sets 'A' and 'B' of the AURORA-2 corpus. Test set A comprises 1 clean and 24 noisy subsets, containing four noise types (subway, car, babble, exhibition hall) at 6 SNR levels (clean, 20,15, $10,5,0$ and $-5 \mathrm{~dB}$ ). Test set $\mathrm{B}$ contains four different noise types (restaurant, street, airport, train station). We defined two sets of testing data. The first set is the full testset, in which each noisy subset contains 1001 utterances with one to seven digits ' $0-9$ ' or 'oh'. The second testset is formed by the same random, representative subset of $10 \%$ of the utterances (i.e. 400 utterances per SNR level) used in [5]. In the results, the results of the four noise types are averaged and we display the results for clean speech, $-5 \mathrm{~dB}$, and the average over the $20-0 \mathrm{~dB}$ range.
The speech and noise dictionaries were created using the two-step procedure described in [3]. First, from each noisy utterance in the multi-condition AURORA-2 training set two segments were randomly selected. The segments were allowed to overlap and no effort was made to exclude silence frames from the exemplars. For these segments the underlying clean speech and noise originally used for creating the noisy speech were extracted from their respective spectrograms and added to the speech and noise dictionaries. This resulted in initial speech and noise dictionaries consisting of 16880 exemplars. From the initial noise dictionary, a noise dictionary of $K=4000$ exemplars was randomly selected. We created two speech dictionaries: the first consists of $J=4000$ speech exemplars randomly extracted from the initial dictionary, and the second consists of $J=10000$ randomly selected speech exemplars. The 4000 exemplar dictionary is a subset of the 10000 exemplar dictionary. Note that the multi-condition training set of AURORA-2 contains the same noise types as test set A.

Acoustic feature vectors used in the compositional model consisted of MEL frequency magnitude spectrograms, spanning $B=23$ bands with a frame length of $25 \mathrm{~ms}$ and a frame shift of $10 \mathrm{~ms}$. We used exemplars spanning $300 \mathrm{~ms}$, thus $T=30$ frames. A sliding window approach was used with windows shifted by a single frame (i.e. $10 \mathrm{~ms}$ ). Digits were described by $16 \mathrm{HMM}$ states with an additional 3-state silence word, resulting in a 179 dimensional state-space. Each frame in each exemplar was annotated using a HMM state label obtained through forced alignment with the canonical transcription obtained with the GMM-based recognizer also employed for use with FE. The SC and FE speech decoding systems were implemented in MATLAB and finding the sparse representation was accelerated with GPU using the GPUmat [8] toolbox; we refer the reader to $[3,5]$ for further implementation details.

Experiments with FE and multi-stream SC-FE employed a GMM-based recognizer operating on MFCC features, derived from the mel-spectral features used in the compositional model. Here, we used 13 static MFCC features along with their delta and delta-delta time derivatives resulting in a 39 dimensional feature vector. The MFCC features were mean and variance normalized on a per-utterance basis. The acoustic model consisted of 64 Gaussians with diagonal covariance per HMM-state and was trained using the EM algorithm. In the multi-stream system, the SC-stream exponent $\alpha$ was kept fixed at 1 and the GMM-stream exponent $\beta$ was set to 0.33 after non-exhaustive tuning on the multi-condition training set.

\section{Experiments and results}

\subsection{Advances in exemplar-based robust ASR}

In this experiment, we investigate the cumulative effect of using some of the advances proposed in [4-6]. Below, we list the steps we consider in this work: 
Table 2: WER obtained on AURORA-2 obtained with FE recognition as a function of SNR. The settings described on each row describe various combinations of the used training data (clean or multi-condition), and whether or not FE was applied to the features during training and/or testing.

\begin{tabular}{|c|c|c||c|c|c|c|c|}
\hline \multirow{2}{*}{ trainingdata } & \multirow{2}{*}{} & \multirow{2}{*}{ testing } & training & clean & \multicolumn{2}{|c|}{ test set A $20-0$} & \multicolumn{2}{c|}{ test set B } \\
& no FE & no FE & 0.2 & 16.0 & 78.6 & 15.0 & 77.3 \\
clean & no FE & FE & 0.1 & 4.8 & 33.1 & 7.2 & 53.3 \\
& FE & FE & $\mathbf{0 . 0}$ & 4.7 & 32.8 & 7.0 & 52.1 \\
\hline \hline \multirow{3}{*}{ multi } & no FE & no FE & 0.9 & 7.2 & 62.8 & 7.6 & 62.7 \\
& no FE & FE & 0.9 & 7.3 & 37.8 & 9.0 & 55.9 \\
& FE & FE & 0.5 & $\mathbf{3 . 5}$ & $\mathbf{2 9 . 6}$ & $\mathbf{5 . 8}$ & $\mathbf{4 7 . 5}$ \\
\hline
\end{tabular}

SC/FE Baseline The SC and FE baseline systems are the systems described in [3]. In short, the baseline SC and FE systems applied 200 iterations of the multiplicative update described in [3] and used a sparsity value of $\lambda=0.65$ for speech exemplars and $\lambda=0$ for noise exemplars. The acoustic features and acoustic model used for FE evaluation are different, however, and are described in Section 3.

Sparsity In [5] it was shown that the recognition accuracy of $\mathrm{SC}$ in both clean and noisy conditions improves if both speech and noise sparsity are jointly tuned in the range $[0,5]$. In this work, we investigate the impact of changing the speech and noise sparsity from 0.65 and 0 , respectively, to 1.5 and 1 . These values are based on the same type of grid search as carried out in [5], but applied to a larger set of randomly selected noisy utterances from the multi-condition train set.

Number of iterations In [5], is was shown that higher number of iterations of the multiplicative updates used to find a sparse representation of the noisy speech may be beneficial. In this step, we investigate to what extend the results improve if we increase the number of iterations from 200 to 600 .

Number of exemplars Originally, $J=4000$ exemplars were used to represent the clean speech. In [5] and [6] experiments were carried out using larger dictionary sizes. In this step, we investigate the impact of using a speech dictionary containing $J=10000$ exemplars.

Artificial noise dictionary In [4] it was proposed to use an artificial noise dictionary to match unseen noise types. This artificial noise dictionary consists of $B$ exemplars, each described by an all-zeros exemplar except for a single active (constant) frequency band. In [5] it was shown that the combination of an artificial noisy dictionary with an exemplar-based noise dictionary results in a system robust on both matched and mismatched noise. In this step we add the artificial noise dictionary.

Noise sniffing In [5] it was proposed to extract the first few frames from the noisy speech to be used as noise exemplar. In this work, we use a small variation of that approach: In additional to a noise exemplar 'sniffed' from the first $T$ frames of the noisy utterance, we include $T-1$ copies of it, each circular shifted by a single frame. This provides a degree of shiftinvariance to the extracted noise exemplar, hopefully improving the match later in the noisy utterance. After this step, the noise dictionary consists of $K+B+T=4000+23+30=4053$ noise exemplars.

In order to reduce the experimentation time, the effect of these steps were evaluated on the reduced test set described in Section 3. In Table 1 we report the WER obtained with both SC and FE (evaluated using an acoustic model trained on unprocessed clean speech). We can observe that for SC, each step yields fairly large WER reductions and the lowest WERs are obtained when all advances are combined, i.e., after addition of the extra noise exemplars. Although not immediately apparent from the experiments presented in [5] and the results in Table 1, further experimentation has shown that some steps indirectly influence the later steps: for example, increasing the number of iterations makes the increase of speech dictionary size more effective.

For FE, the effect of each step is less noticeable due to the high baseline accuracy on clean speech. Also, there is a more noticeable trade-off between accuracy on matched noise types (test set A) and mismatched noise types (test set B) - the use of additional noise exemplars leads to a small increase in WER. When comparing the WERs obtained with SC and FE it can be observed that SC performs better on noisy mismatched speech (test set B), but FE performs better at high SNRs and on noisy speech corrupted by matched noise types. This result is markedly different from the result obtained in [3], where FE only outperformed SC at high SNRs. The difference can be attributed to the use of normalized MFCC features, a larger number of Gaussians per state, and the full re-training of the acoustic model rather than the single-pass retraining employed in [3].

\subsection{Acoustic model training for $\mathbf{F E}$}

The final system of the previous experiment achieved the lowest WERs on noisy speech corrupted by mismatched noise types, and is used as a starting point in this experiment. Here, we investigate several aspects of the acoustic model training used in the GMM-based recognizer operation on FE-processed features. First, we investigate the impact of using FE on the features used for training in order to reduce the training-test mismatch. Second, we investigate to what extend the results improve when we use the multi-condition training set rather than the clean training set. Since the noisy speech in the multicondition training set was already used to form the exemplar noise dictionary, the use of multi-condition training data is a valid choice. Moreover, most ASR systems deployed in practice are multi-condition trained [9]. For reference, we also include a do-nothing ASR baseline in which neither the features used in training nor in testing are processed with FE. As in the previous experiment, we employed the reduced test set described in Section 3.

In Table 2 we can observe that the use of FE yields a substantial improvement over the non-robust baseline and that the WER decreases if the mismatch between training and testing is reduced by processing the noisy utterances used for training with FE. We can further observe that the use of multi-condition training data improves the noise robustness of the FE system, at the cost of a higher WER on clean speech. What may be surprising however, is that the use of FE during training and testing improves the WER on clean speech beyond the baseline accuracy. When using multi-condition training data, this can be explained by noting that the average SNR of the multi-condition training data (about $5 \mathrm{~dB}$ ) has increased due to the application of FE, resulting in a better match on clean speech. When using clean speech training data, however, a small although not signif- 
Table 3: WER obtained on AURORA-2 as a function of SNR. The best performing SC and FE systems are shown, as well as the multistream combination of SC and FE. The abbreviations $c l n$ and $m l t$ refer to the use of clean and multi-condition training data, respectively. Additionally, baseline results and results obtained with two alternative noise robust ASR systems described in literature are shown.

\begin{tabular}{|c||c|c|c|c|c|}
\hline \multirow{2}{*}{ method } & \multirow{2}{*}{ clean } & \multicolumn{2}{c|}{ test set $A$} & \multicolumn{2}{c|}{ test set $B$} \\
& $20-0$ & -5 & $20-0$ & -5 \\
\hline \hline Baseline (cln) & 0.3 & 16.9 & 77.2 & 15.9 & 77.1 \\
Baseline (mlt) & 0.9 & 7.5 & 61.4 & 7.9 & 64.2 \\
\hline \hline SC & 3.5 & 7.2 & 35.7 & 9.3 & 49.8 \\
FE (cln) & $\mathbf{0 . 2}$ & 5.0 & 34.1 & 7.7 & 53.9 \\
FE (mlt) & 0.4 & 3.6 & 30.4 & 6.1 & 50.8 \\
\hline \hline SC + FE (cln) & 0.4 & 4.1 & 28.7 & 5.6 & 45.8 \\
SC + FE (mlt) & 0.5 & $\mathbf{3 . 1}$ & $\mathbf{2 5 . 6}$ & 5.0 & $\mathbf{4 3 . 7}$ \\
\hline \hline ESSEM & - & 4.4 & - & $\mathbf{4 . 7}$ & - \\
CMLLR & 0.4 & 6.4 & 57.7 & 5.7 & 53.8 \\
\hline
\end{tabular}

icant decrease in WER can also be observed. In [10], a similar effect was observed and there it was explained that this is due to FE using the speech dictionary to map the observed speech back to the speech manifold observed during training.

\subsection{Hybrid recognition and comparison to state-of-the-art}

In this final experiment, we apply the best performing SC and FE systems, i.e., the final SC system from Section 4.1 and the clean and multi-condition trained FE systems (with FE applied to both train and testsets) from Section 4.2 to the full AURORA2 testset. Moreover, we investigate to what extent the results improve when we combine these systems at the probability level using the procedure described in Section 2.3.

The results of the experiments with SC, FE and SC-FE are shown in Table 3. Additionally, the do-nothing baseline described in Section 4.2 is shown for clean (cln) and multicondition (mlt) training, as well as the results obtained with two recent alternative robust ASR methods that exploit multicondition training data: 1) ESSEM, which works by first constructing a large set of gender\&environmental dependent GMM's, and selecting and transforming these sets at runtime [11]. To the best of our knowledge, the $20-0 \mathrm{~dB}$ average WERs reported in [11] are the lowest to-date on AURORA-2. 2) CMLLR, which relies on a method called factored adaptation to jointly compensate for speaker and environmental mismatch using a cascade of Constrained Maximum-Likelihood Linear Regression (CMLLR) transforms that separately compensate for the environment and speaker variability [9]. Note that the results obtained with this method are averages over only (the last) three noise types described in Section 3, with the first noise type used for adaptation. The WERs obtained with SC and FE systems would come out even lower if only considering these three noise types.

The results in Table 3 indicate that although there are small absolute differences between the results obtained on the reduced testsets employed in Sections 4.1 and 4.2 and the results obtained on the full testset, overall there is good agreement of the relative differences between methods. Moreover, the combination of SC and FE into a hybrid multi-stream recognizer yields substantial lower WERs on noisy speech both with clean- and multi-condition trained acoustic models. In fact, the results obtained with the multi-condition trained hybrid system on mismatched noise types (test set B) are competitive with the best results published to date $(5.0 \%$ for SC-FE vs. $4.7 \%$ for ESSEM), and the $3.1 \%$ WER on matched noise types (test set A) is substantially better than the best results to date.

\section{Conclusions and future work}

In this work we presented three lines of research. First, we investigated the cumulative contribution of a number of $\mathrm{SC}$ advances recently proposed in literature and showed that these advances do combine to yield ASR system that is robust to both matched and mismatched noise types. Second, we improve upon the results obtained with FE through retraining and multi-condition training of the acoustic models used in a conventional GMM-based recognizer and third, we propose the use of a hybrid multi-stream SC-FE system that combines the two systems at the state probability level. Experiments on AURORA2 showed that a multi-condition trained hybrid SC-FE achieves an impressive $3.1 \%$ and $5.0 \%$ average WER on matched and on mismatched noise types, respectively, which is among the best results ever published on this dataset.

Future work focuses on further improving the noise robustness on mismatched noise types, evaluations on large vocabulary speech databases and on addressing non-additive signal corruptions such as channel effects and reverberation.

\section{Acknowledgments}

This research was funded by IWT-SBO grant 100049 (ALADIN).

\section{References}

[1] P. Moreno, B. Raj, and R. Stern, "A vector taylor series approach for environment-independent speech recognition," in Proc. ICASSP, 1996.

[2] M. Cooke, P. Green, L. Josifovksi, and A. Vizinho, "Robust automatic speech recognition with missing and unreliable acoustic data," Speech Communication, vol. 34, no. 3, pp. 267-285, June 2001.

[3] J. F. Gemmeke, T. Virtanen, and A. Hurmalainen, "Exemplar-based sparse representations for noise robust automatic speech recognition," IEEE Transactions on Audio, Speech and Language processing, vol. 19, no. 7, pp. 2067-2080, 2011.

[4] J. F. Gemmeke and T. Virtanen, "Artificial and online acquired noise dictionaries for noise robust ASR," in Proc. Interspeech, 2010.

[5] J. F. Gemmeke, A. Hurmalainen, T. Virtanen, and Y. Sun, "Toward a practical implementation of exemplar-based noise robust ASR," in Proc. EUSIPCO, 2011, pp. 14901494.

[6] J. F. Gemmeke and Hugo Van hamme, "An Hierarchical Exemplar-based Sparse Model of Speech, with an Application to ASR," in Automatic speech recognition and understanding Workshop, 2011.

[7] Y. Sun, J. F. Gemmeke, B. Cranen, L. ten Bosch, and L. Boves, "Using a DBN to integrate Sparse Classification and GMM-based ASR," in Proc. Interspeech, 2010.

[8] "GPUmat: GPU toolbox for MATLAB," Online: http://gp-you.org/, 2010.

[9] M. L. Seltzer and A. Acero, "Factored adaptation for separable compensation of speaker and environmental variability," in Proc. ASRU, 2011.

[10] T. N. Sainath, B. Ramabhadran, D. Nahamoo, D. Kanevsky, and A. Sethy, "Exemplar-Based Sparse Representation Features for Speech Recognition," in Proc. Interspeech, 2010.

[11] Y. Tsao, J. Li, and C.-H. Lee, "Ensemble speaker and speaking environment modeling approach with advanced online estimation process," in Proc. ICASSP, 2009, pp. 3833-3836. 